INPLASY

PROTOCOL

To cite: Yang et al. The

influence of different doses of aspirin on pregnancy outcome. Inplasy protocol 2020100107. doi:

10.37766/inplasy2020.10.0107

Received: 27 October 2020

Published: 28 October 2020

Corresponding author:

Yang Rui

760905005@qq.com

Author Affiliation:

North Sichuan Medical College

Support: No.

Review Stage at time of this submission: The review has not yet started.

Conflicts of interest: No.

\section{The influence of different doses of aspirin on pregnancy outcome}

\author{
Yang, R' Huang, $R^{2}$.
}

Review question / Objective: P (research subjects): pregnant women; I (intervention measures): low-dose aspirin; C (control measures): placebo treatment or blank control; $O$ (outcome indicators): preeclampsia, premature delivery, miscarriage, duration of pregnancy, Low birth weight, stillbirth, perinatal death, postpartum hemorrhage, etc.; $\mathbf{S}$ (research design plan): randomized controlled study (RCT).

Condition being studied: At present, the doses of aspirin used in studies related to the prevention of preeclampsia are 50 $\mathrm{mg}, 60 \mathrm{mg}, 75 \mathrm{mg}, 80 \mathrm{mg}, 81 \mathrm{mg}, 100 \mathrm{mg}$, and $150 \mathrm{mg}$. Comparing the above application doses, in 2014, the US Preventive Services Task Force (USPSTF) recommended lowdose aspirin $(81 \mathrm{mg} / \mathrm{d})$ after 12 weeks of pregnancy in the lowdose aspirin prevention clinical guidelines for preeclampsia, and the American College of Obstetrics and Gynecology recommended the first three pregnancy Start taking low-dose aspirin $(60-80 \mathrm{mg} / \mathrm{d})$ at the end of the month. The World Health Organization recommends that high-risk women start taking low-dose aspirin $(75 \mathrm{mg} / \mathrm{d})$ from $12-20$ weeks of pregnancy. National Institute of Health and Clinical Optimization (NICE) It is recommended that women at high risk of preeclampsia take aspirin $(75 \mathrm{mg} / \mathrm{d})$ from the 12 th week of pregnancy to delivery. There are still controversies about the specific administration time and dosage of aspirin.

INPLASY registration number: This protocol was registered with the International Platform of Registered Systematic Review and Meta-Analysis Protocols (INPLASY) on 28 October 2020 and was last updated on 28 October 2020 (registration number INPLASY2020100107).

\section{INTRODUCTION}

Review question / Objective: P (research subjects): pregnant women; I (intervention measures): low-dose aspirin; C (control measures): placebo treatment or blank control; 0 (outcome indicators): preeclampsia, premature delivery, miscarriage, duration of pregnancy, Low birth weight, stillbirth, perinatal death, postpartum hemorrhage, etc.; S (research design plan): randomized controlled study (RCT). 
Rationale: This study intends to understand the effects of aspirin on pregnancy complications through the analysis of previous relevant literature, and to evaluate the dose of aspirin recorded in the existing literature to further explore the scope and dosage of aspirin during pregnancy.

Condition being studied: At present, the doses of aspirin used in studies related to the prevention of preeclampsia are $50 \mathrm{mg}$, $60 \mathrm{mg}, 75 \mathrm{mg}, 80 \mathrm{mg}, 81 \mathrm{mg}, 100 \mathrm{mg}$, and $150 \mathrm{mg}$. Comparing the above application doses, in 2014, the US Preventive Services Task Force (USPSTF) recommended lowdose aspirin $(81 \mathrm{mg} / \mathrm{d})$ after 12 weeks of pregnancy in the low-dose aspirin prevention clinical guidelines for preeclampsia, and the American College of Obstetrics and Gynecology recommended the first three pregnancy Start taking lowdose aspirin $(60-80 \mathrm{mg} / \mathrm{d})$ at the end of the month. The World Health Organization recommends that high-risk women start taking low-dose aspirin $(75 \mathrm{mg} / \mathrm{d})$ from 12-20 weeks of pregnancy. National Institute of Health and Clinical Optimization (NICE) It is recommended that women at high risk of preeclampsia take aspirin $(75 \mathrm{mg} / \mathrm{d})$ from the 12th week of pregnancy to delivery. There are still controversies about the specific administration time and dosage of aspirin.

\section{METHODS}

Search strategy: Search keywords: aspirin, acetylsalicylic acid, ascitesmin, preeclampsia, preterm birth, pregnancy, pregnancy complications, randomized controlled trials, randomized controlled studies.

Participant or population: Pregnant women.

Intervention: Low-dose aspirin.

Comparator: Placebo treatment or blank control.

Study designs to be included: Randomized controlled study.
Eligibility criteria: 1) Literature inclusion criteria: P (research subjects): pregnant women; I (intervention measures): low-dose aspirin; C (control measures): placebo treatment or blank control; $O$ (outcome indicators): pre-eclampsia, preterm birth , Miscarriage, duration of pregnancy, low birth weight, stillbirth, perinatal death, postpartum hemorrhage, etc.; $S$ (research design): randomized controlled study (RCT). 2) Document exclusion criteria: only complete publications, English or Chinese documents are considered. Two reviewers independently read the title and abstract of the literature. If necessary, read the complete report and bibliography. According to the literature inclusion criteria, independently select the literature to be included, and then compare each other. If there is a difference, please discuss with the third reviewer. Decided. When multiple reports involve the same subjects, test methods, and outcome indicators, the most complete report is selected.

Information sources: By searching existing literature databases such as the US National Library of Medicine medline, Dutch Medical Abstracts (embase), Cochrane Library, Web of Science, ClinicalTrials, Science Citation Index (SCI), Chinese Biomedical Literature Database (CBM), China Knowledge Network (CNKI), Wanfang Data Knowledge Service Platform, etc., and manually search all references including research.

Main outcome(s): 1. Through the analysis of previous studies of aspirin mainly in preeclampsia, in order to find out whether aspirin is suitable for other pregnancy complications in addition to reducing the risk of preeclampsia. 2. Compare the current research doses of aspirin in order to find out whether increasing or decreasing the dose of aspirin under the current recommended dose of aspirin is more beneficial in reducing complications during pregnancy.

Additional outcome(s): No. 
Data management: Using an Excel spreadsheet designed in advance, two reviewers independently read all the included literature and extract relevant data, hide the authors, units and published journals of the research from the reviewers, and use Engauge Digitizer software for the extraction of graphics data, and the original text if necessary The author contacted to obtain complete data as much as possible. When two evaluators disagree, the third evaluator is asked to discuss and decide.

Quality assessment / Risk of bias analysis: Use Review Manager 5.3 to assess the risk of bias for the included literature.

Strategy of data synthesis: Use Review Manager 5.3 to calculate the relative risk or weighted mean difference (WMD) and 95\% confidence interval $(95 \% \mathrm{Cl})$ of the main outcome indicators, and perform subgroup analysis on different doses of aspirin. If you want to change the current question, then Record and use sensitivity analysis. When the heterogeneity between studies is small $(12 \leq 50 \%)$, the fixed effects model is used, and the heterogeneity is large (12>50\%) using the random effects model.

Subgroup analysis: Subgroup analysis of different doses of aspirin.

Sensibility analysis: No.

Language: No restriction.

Country(ies) involved: China.

Other relevant information: No.

Keywords: Aspirin, pregnancy complications, effects.

Dissemination plans: No.

Contributions of each author:

Author 1 - Yang Rui.

Author 2 - Huang Renying. 